\title{
Ergebnisse des Seminars „10 Jahre Gewaltschutzgesetz - neue Herausforderungen“
}

\author{
Dagmar Freudenberg \\ Vorsitzende der Kommission Strafrecht des djb; Staatsanwältin, \\ Göttingen/Hannover
}

In seinem Seminar „10 Jahre Gewaltschutzgesetz - neue Herausforderungen " hat der djb in Bonn im Oktober die Ergebnisse der Länderumfrage 2011 zur Umsetzung des Gewaltschutzgesetzes vorgestellt. Die von allen 16 Bundesländern übermittelten Antworten machen deutlich, dass bei Schutz und Unterstützung der zumeist weiblichen Opfer häuslicher Gewalt durch die Implementierung von Aktionsplänen und die Einrichtung von Koordinierungsstellen bereits deutliche Fortschritte erzielt wurden.

\section{Internationaler Fokus}

Genauso eindeutig steht nach dem Ergebnis der Umfrage allerdings auch fest: Häusliche Gewalt ist keineswegs aus der Gesellschaft verschwunden. Die Bekämpfung häuslicher Gewalt muss deshalb auf allen Ebenen konzentriert weitergeführt werden. Auf internationaler, europäischer Ebene hat Deutschland die Europaratskonvention gegen Gewalt gegen Frauen und häusliche Gewalt 2011 zwar gezeichnet. Ratifizierung und Umsetzung stehen jedoch noch aus und müssen vollzogen werden, wie die Direktorin des Instituts für Menschenrechte, Prof. Dr. Beate Rudolf, erläuterte. Der djb wird prüfen, welche in der Konvention vorgesehenen Maßnahmen der Gesetzgebung, des Schutzes der Opfer und der Inverantwortungnahme der Täter noch fehlen. Die Umsetzung in entsprechenden Aktionsplänen wird der djb unterstützen.

Eine Maßnahme, die auch in der Europaratskonvention vorgesehen ist, kann mit dem Ergebnis der Länderumfrage des djb schon jetzt eindeutig als noch nicht umgesetzt belegt werden:

Die statistische Erfassung von Gewaltschutzverfahren im Zivilrecht erfolgt in den einzelnen Bundesländern sehr unterschiedlich. Dies betrifft sowohl die Frage nach der Häufigkeit der unterschiedlichen Verfahren und der jeweiligen Verfahrensdauer, aber auch die verfahrensrechtliche Verbindung von Umgangs- und Sorgerechtsverfahren mit Gewaltschutzverfahren und die Häufigkeit der Durchführung einer mündlichen Verhandlung auf Antrag des Antragsgegners. Zu der für den Schutz vor Eskalation wichtigen Verfahrensdauer konnten nur einige Bundesländer nähere Angaben machen. Meist wurde auf die Möglichkeit der Durchführung eines einstweiligen Anordnungsverfahrens und dessen typischerweise kurze Verfahrensdauer hingewiesen.

Im Strafrecht werden nicht nur bundesweit, sondern teilweise auch landesweit unterschiedliche Definitionen berichtet. Teilweise wurde überhaupt keine Definition genannt. Als häusliche Gewalt wird sowohl Partnergewalt bearbeitet, als auch sehr weitgehend jede Gewalt gegenüber Familienangehörigen oder sogar Gewalt im Kontext von (Senioren-)Wohngemeinschaften, teilweise wird eine (bestehende oder frühere) häusliche Gemeinschaft verlangt, überwiegend wird dies jedoch nicht als Voraussetzung angesehen. Auch die der Einordnung als häusliche Gewalt zugrundeliegenden Deliktsformen unterscheiden sich stark. ${ }^{1}$ Um eine Vergleichbarkeit der Bearbeitungsweisen und Belastungen zu erleichtern ist eine bundesweit einheitliche Definition jedoch erste Voraussetzung. Das Fehlen einer einheitlichen begrifflichen Arbeitsgrundlage führt zu nicht vergleichbarem Zahlenmaterial. National und international vergleichbar erhobene Zahlen sind aber notwendige Grundlage einer gelingenden Rechts-, Jugend-, Sozialund Sicherheitspolitik. Der djb wird sich deshalb insbesondere die Mitarbeit an der Entwicklung eines leistungsstarken und überstaatlich wie innerstaatlich vergleichbaren Monitorings zur Aufgabe machen. Eine einheitliche Definition häuslicher Gewalt in Deutschland und Europa für Polizei, Justiz und Opferunterstützungseinrichtungen kann auch für eine angemessene Bewertung der Arbeit der Professionen und die Vergleichbarkeit der Ergebnisse der Arbeit gegen häusliche Gewalt in Deutschland genutzt werden.

\section{Maßnahmen im zivilgerichtlichen Bereich}

Die Reform des Gewaltschutzgesetzes 2009, durch die eine einheitliche Zuständigkeit des Familiengerichts eingeführt wurde, hat sich nach dem Ergebnis der Umfrage bewährt, auch wenn damit nun auch Verfahren bearbeitet werden müssen, die als Miet- und Nachbarstreitigkeiten nicht zwangsläufig zur Zuständigkeit des Familiengerichts gehören. Die Richterschaft wertete als positiven Effekt dieser Gesetzesänderung, dass aufgrund der jetzt geltenden Zuständigkeitsregelung kein Zeitverlust wegen Abklärung der Zuständigkeit mehr zu verzeichnen ist. Genau diese Gesetzesänderung war seinerzeit eine Forderung des djb, deren Umsetzung offensichtlich zu einer Entlastung der Gerichte und zu einer Beschleunigung geführt hat.

Die auch vom djb begrüßte Möglichkeit der getrennten Anhörung von Opfer und Täter wird in den Familiengerichten dagegen bedauerlicherweise nur in Einzelfällen genutzt. Dies liegt nach den Antworten der Länder sowohl an fehlenden Anregungen durch das Opfer als auch daran, dass Gerichte eine höhere Sachverhaltsaufklärung von der Durchführung einer gemeinsamen Anhörung erwarten. Die fehlende Antragstellung dürfte in der Unkenntnis der Opfer und Opfervertretungen über diese rechtliche Möglichkeiten begründet sein. Weitere Aufklärungsarbeit ist hier offenbar dringend notwendig.

1 S. dazu den Beitrag „Zehn Jahre Gewaltschutzgesetz" von Susanne Köhler in diesem Heft, S. 11. 


\section{Strafrechtliche Verfolgung häuslicher Gewalt}

Schon anlässlich der Verabschiedung des Gewaltschutzgesetzes hat der djb darauf hingewiesen, dass die strafrechtliche Verfolgung häuslicher Gewalt intensivere Ermittlungsarbeit, phänomenbezogenes interdisziplinäres Fachwissen und Vernetzungsstrukturen erfordert. Deshalb wurde seitens des djb stets die Einrichtung von Sonderdezernaten gefordert. Zur flächendeckenden Einrichtung von Sonderdezernaten „Häusliche Gewalt“ bei den Staatsanwaltschaften können gegenüber der Umfrage des djb aus dem Jahr 2004 indessen nur wenige Änderungen festgestellt werden. Den Antworten der Bundesländer ist zu entnehmen, dass die in der früheren Umfrage schon beschriebene Einrichtung von Sonderdezernaten zwar noch etwas vorangeschritten, aber doch immer noch nicht flächendeckend umgesetzt ist. Gerade große Bundesländer teilen mit, dass nur teilweise Sonderdezernate bestehen. Mancherorts wird in Beantwortung der Frage auf „Ansprechpartner“ oder „Koordinatoren“ für Fälle häuslicher Gewalt mit Zuständigkeiten sowohl innerhalb der Behörde als auch für Kontakte nach außen hingewiesen. Diese Ansprechpartner/innen können jedoch keine adäquate Alternative zur Einrichtung von Sonderdezernaten bieten. Sonderdezernate sind gerade deswegen so erfolgreich, weil im Rahmen der Spezialisierung auf das Gebiet häusliche Gewalt, auf die damit verbundenen strafrechtlichen, strafprozessualen und psychosozialen Fragestellungen ein Sachverstand gebündelt wird, der sich insbesondere bei der praktischen Arbeit im Einzelfall sinnvoll auswirkt. Allein von kollegialer Kommunikation mittels Ansprechpersonen nach innen und nach außen ist dies nicht zu erwarten. Die auch vom djb immer wieder geforderte Beteiligung der Staatsanwaltschaften an der Vernetzung ist dagegen weitgehend umgesetzt.

\section{Umsetzung des Gewaltschutzgesetzes und Vernetzung der Professionen}

Als Ergebnis zum Bereich der Umsetzung des Gewaltschutzgesetzes durch Vernetzung kann festgestellt werden, dass in nahezu allen Ländern insbesondere auch die mitbetroffenen Kinder in den Fokus der Bekämpfung häuslicher Gewalt ge- nommen wurden und deshalb die Jugendämter und andere Institutionen für Kinder mit beteiligt werden. Weitere Professionen im Gesundheitsbereich sind dagegen noch nicht überall einbezogen.

\section{Fazit}

Internationale Vorgaben müssen durch Ratifizierung der Europaratskonvention geprüft und umgesetzt werden.

Die bereits begonnenen strukturierten Maßnahmen zur Intervention müssen weiter entwickelt, intensiviert und möglichst flächendeckend angeboten werden.

Insbesondere muss die Finanzierung der Frauenhäuser, der Beratungsstellen und der Täterarbeit sichergestellt werden.

Schließlich kann als Ergebnis der Länderumfrage 2011 des djb festgehalten werden, dass über die Intervention hinaus die Prävention nicht nur durch Ausbau der Täterarbeit, sondern auch durch Sensibilisierung in verschiedenen gesellschaftlichen Bereichen wie der Schule, der Nachbarschaft und in den Betrieben dringend verstärkt werden muss.

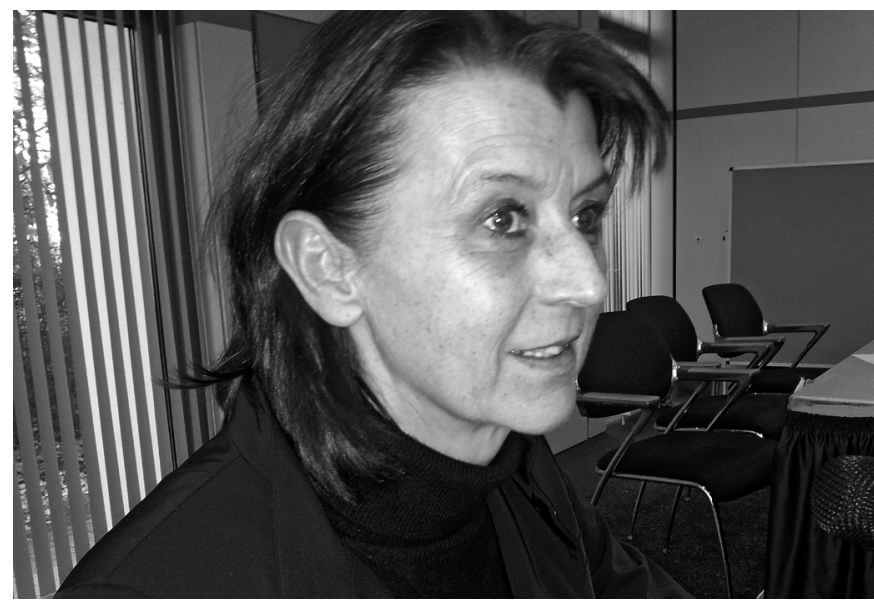

A Prof. Dr. Luise Greuel, Rektorin der Hochschule für Öffentliche Verwaltung, Bremen, stellte den Umgang mit Hochrisikofällen bei häuslicher Gewalt und Stalking dar. (Ihr Vortrag, der großes Interesse fand, wird in Heft 2/2013 abgedruckt.) 